\title{
Efficient Diagnosing Method for Heart Disease Using Deep Learning
}

\author{
Jayanthi $\mathrm{S}^{\mathrm{a}, 1}$, Hajara FathimaS ${ }^{\mathrm{b}}$, IndiraniG ${ }^{\mathrm{c}}$, Dr.C.R.ReneRobin ${ }^{\mathrm{d}}$ \\ ${ }^{a}$ Assistant Professor, Dept of CSE, Agni College of Technology, Chennai \\ ${ }^{b, c} U G$ Student, Deptof CSE, Agni College of Technology, Chennai \\ ${ }^{d}$ Professor, Dept of CSE, Sri Sairam Engineering College, Chennai
}

\begin{abstract}
Heart Disease(HD) is one of the most serious health issue that attacks people age from 65 and older and has symptoms are palpitations, loss of conscious ,abnormal heart beats and it also can attack younger people who has going through lots of stress, over weight and chest pain and so on. Diagnosing heart disease manually is less efficient and mostly not accurate. Machine Learning (ML) helps efficiently in early prediction of Heart Attack. In this paper we have used LSTM (Long Short Term Memory) a Deep Learning Technique to diagonise heart attack. Diagnosing is complicated as it is important task, itneeds to be executed accurately and efficiently. This system helps in prediction of HD which used the supervised learning that has low computation.
\end{abstract}

Keywords. LSTM, Neural network, Heart disease Prediction.

\section{Introduction}

Heart disease is rising around worldwide. In earlier days, the older people only get heart disease but now a days even young teenagers also get affected by heart attack ,For example, In U.S more children getting affected by Heart Disease which includes congenital heart disease, Arrhythmias to prevent this we have to predict the disease in early stages Incidence of heart disease is set to rise, with an aging global population. The heart disease increasing in adults as well as youngsters which leads to aim of better prevention and treatment of heart disease. We build the system which gives suggestions for common treatment for heart attack. Heart disease can also be prevented by food control, stress control. For example, Foods like Leafy green vegetables, whole grains, Avocados, Walnuts, etc.., This paper deals with segment I describes introduction, segment II describes related work, segment III presents methodology, segment IV describes module description, segment $\mathrm{V}$ deals with analysis and segment VI gives conclusion and future work and finally, the reference.

jayanthi S, Assistant Professor, Dept of CSE, Agni College of Technology, Chennai, India. Email:jayanthi.cse.act@edu.in ${ }^{1}$ 


\section{Related Work}

R.Miclo et al. (2015) Real time location system based process mining uses process mining tool that automatically saves the actions in accordance to patients locations in the service. The process mining model does not provide accurate, detailed enough for upcoming research. It has drawbacks that consumes lot of time and may cause interpretations. Min Chen et al.(2017) have demonstrated Disease Prediction by Machine Learning over big data from HealthCare Communities, explain how to use big data in disease prediction. Big data helps ito accurate prediction of disease with patient system is level of accuracy is decreasing when medical data is incomplete and has no voice pathology information. Syed Umar Amin et al. (2019) have demonstrated Cognitive Smart Healthcare for pathology Detection and Monitoring they propose a cognitive healthcare model that combines IOT-cloud technologies for pathology detection and classification. This method have $87 \%$ accuracy, we need to increase efficiency by adding new deep learning technique. This system helps to provide high quality health care in real-time and it using sensor readings that allows to find patient state. Anand Sharma et al. (2014) have demonstrated Emerging Application of Data Mining for Healthcare Management, has higher quality of health care, high performance, low-support, low-confidence, they showed a difficult review of the different research. They built system which provide quality healthcare resource management and helps to diagnosis and treatment of various disease. Arianna Dagliati et al.( 2014) have illustrate the technique to identify cardiovascular disease related to multidisciplinary tool to manage patient clinical course .The main goal of their work is to derive health care pathways .It also allows to gain understanding T2D patients care.

\section{Methodology}

In the proposed system, we choose LSTM among various algorithm. LSTM is a deep learning technique which helps to overcome data loss while predicting time series data , as it provides a best solution to vanishing and exploding gradients compare to RNN. It gives effective result in many relevant application like heart disease prediction. This helps to retain the information without loss as it used to accurate prediction of disease.

\section{Module Description}

\section{Module 1 : Exploratory Data Evaluation}

It gives depth understanding of dataset which helps to find the clear view of data .It allows us to use visual and quantitative techniques to determine trends, structure etc. Before making any presumption EDA main purpose to find the pattern of data. It helps to searching and finding out missing data and other mistakes such as errors, anomalies. For example, EDA tool used to create an EDA are python and R language.

\section{Module 2 : Pre-processing}

Scikit Learn preprocessing is a python library which allows to do preprocessing for given dataset. Preprocessing is a term basically means to discover the some lost data in 
the dataset. It is essential to do this process as it have chance to loss the important information that in need to determine heart disease without any delay. We should convert the categorical data into numerical which is the only way for mathematical algorithmic model to understand the data set variables. Since it is quite difficult for machine to process text. In preprocessing it is a important part to split dataset in to two sets called training set and testing set. The python library scikit used to split the dataset according to certain percentage is test_train_split from model_selection.

\section{Module 3 : Feature Engineering}

Feature selection is independent of any machine learning algorithms. It helps more to decrease computational cost by eliminate the less important input variable. Feature is the term represents the column also called variable, feature selection usually based on dependency between the variable, the features of dataset is selected to gives as input to get efficient output. Based on correlation co-efficient that is between -1 to +1 we can have other following method to select feature there are, LDA, Fisher's score and so on.

\section{Module 4 : Prediction}

Prediction can be done by evaluating the model by giving testing dataset which helps to find whether the model is trained well or not. Based on accuracy and speed of the model we can decide. As the splitting of dataset mainly relys on the how large or small the dataset. After the evaluation process is complete, we can test by giving other values.

\section{Analysis}

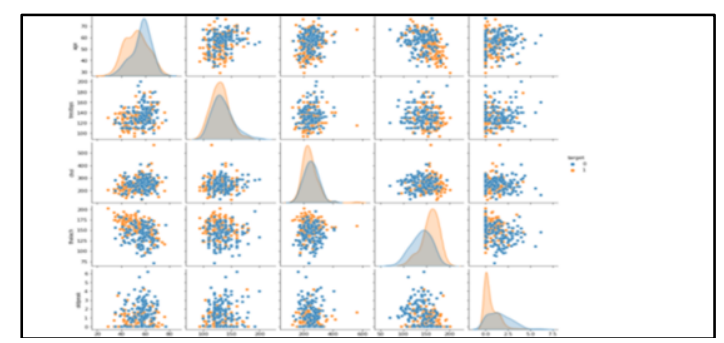

Figure 1. Distribution of highly correlated features over Target variable.

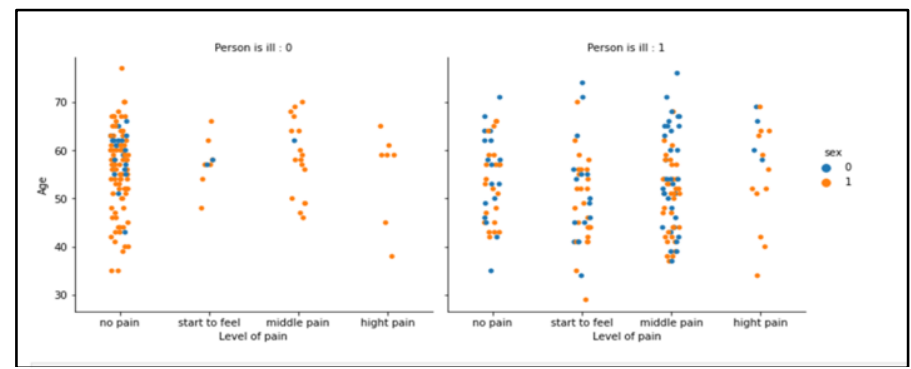

Figure 2.Distribution of ill and not ill person based on Sex ,Age and Level of pain. 


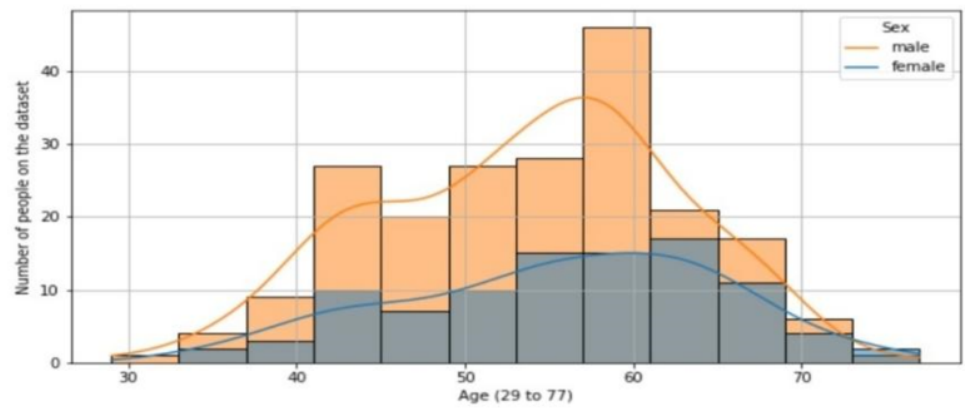

Figure 3. The above histplot represent the number of male and female in between age 29 to 77 in the total number of people on the dataset

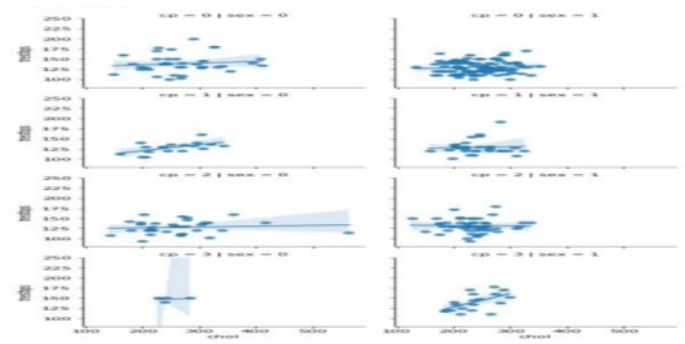

Figure 4. The scatter plot defines relation between trestbpsandcholesterol for each type of chest pain and sex.

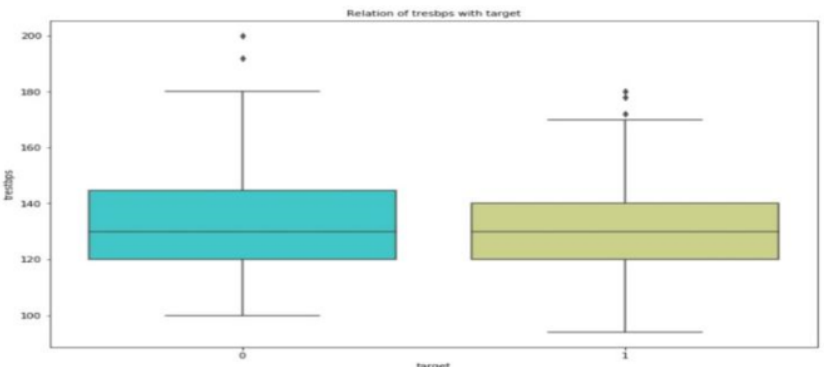

Figure 5.The box plot graph represents between the trestbps and target. 


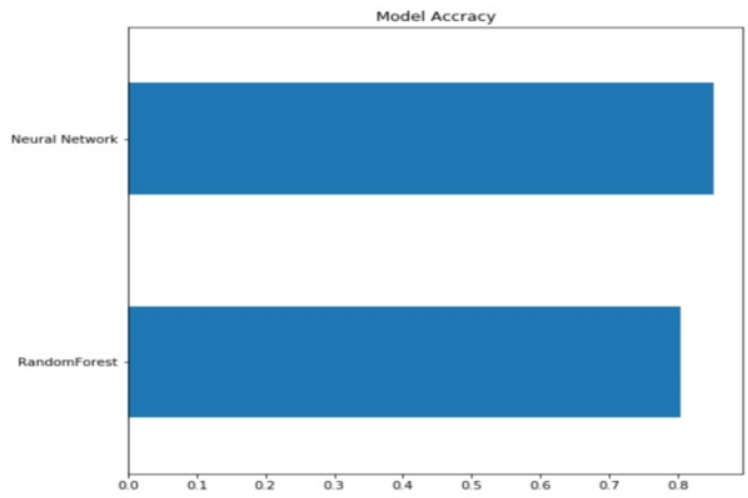

Figure 6. Diagram shows the comparison of Model accuracy between neural network and Random forest, which gives neural network with high accuracy.

\section{Conclusion}

We have developed algorithm with high accuracy which also helps to prevent from missing information. Though it can process well in easily obtainable clinical data. It provides low computation power. In future work will include real-time information for dietary plans and treatment suggestion based on the stages of heart attack. Another future line would be detection of various heart related disease.

\section{References}

[1] M. Durairaj and N. Ramasamy, A comparison of the perceptive approaches for preprocessing the data set for predicting fertility success rate, Int. J. Control Theory Appl., vol. 9, no. 27, pp. $255260,2016$.

[2] Allen.L.A, L.W. Stevenson, K. L. Grady, N. E. Goldstein, D. D. Matlock, R. M. Arnold, N. R. Cook, G. M. Felker, G. S. Francis, P. J. Hauptman,E. P. Havranek, H. M. Krumholz, D. Mancini, B. Riegel, and J. A. Spertus, Decision making in advanced heart failure: A scienti_cstatementfrom the American heart association, Circulation, vol. 125, no. 15, pp. 1928_1952, 2012.

[3] Ghwanmeh.S, A. Mohammad, and A. Al-Ibrahim, Innovative arti_cial neural networks-based decision support system for heart diseases diagnosis, J. Intell. Learn. Syst. Appl., vol. 5, no. 3, 2013, Art. no. 35396.

[4] Lopez-Sendon.J. The heart failure epidemic, Medicographia, vol. 33,no. 4, pp. 363_369, 2011.

[5] P. A. Heidenreich, J. G. Trogdon, O. A. Khavjou, J. Butler, K. Dracup,M. D. Ezekowitz, E. A. Finkelstein, Y. Hong, S. C. Johnston, A. Khera,D. M. Lloyd-Jones, S. A. Nelson, G. Nichol, D. Orenstein, P.W. F.Wilson, and Y. J. Woo, Forecasting the future of cardiovascular disease in the united states: A policy statement from the American heart association, Circulation, vol. 123, no. 8, pp.933_944, 2011.

[6] A. Tsanas, M. A. Little, P. E. McSharry, and L. O. Ramig, Nonlinearspeech analysis algorithms mapped to a standard metric achieve clinicallyusefulquanti_cation of average Parkinson's disease symptom severity, J. Roy. Soc. Interface, vol. 8, no. 59, pp. 842_855, 2011.

[7] S. Nazir, S. Shahzad, S. Mahfooz, and M. Nazir, Fuzzy logic based decision support system for component security evaluation, Int. Arab J.Inf. Technol., vol. 15, no. 2, pp. 224_231, 2018. 
[8] R. Detrano, A. Janosi,W.Steinbrunn, M. P_sterer, J.-J. Schmid, S. Sandhu, K. H. Guppy, S. Lee, and V. Froelicher, International application of a new probability algorithm for the diagnosis of coronary artery disease, Amer.J. Cardiol., vol. 64, no. 5, pp. 304_310, Aug. 1989.

[9] Y. Li, T. Li, and H. Liu, Recent advances in feature selection and its applications, Knowl. Inf. Syst., vol. 53, no. 3, pp. 551_577, Dec. 2017.

[10] J. Li and H. Liu, Challenges of feature selection for big data analytics, IEEE Intell. Syst., vol. 32, no. 2, pp. 9_15, Mar. 2017.

[11] L. Zhu, J. Shen, L. Xie, and Z. Cheng, Unsupervised topic hypergraph hashing for ef_cient mobile image retrieval, IEEE Trans. Cybern., vol. 47,no. 11, pp. 3941_3954, Nov. 2017.

[12] Raschka, Model evaluation, model selection, and algorithm selection in machine learning, 2018, arXiv:1811.12808. [Online]. Available: http://arxiv.org/abs/1811.12808

[13] Palaniappan and R. Awang, Intelligent heart disease prediction system using data mining techniques, in Proc. IEEE/ACS Int. Conf. Comput. Syst.Appl., Mar. 2008, pp. $108 \_115$.

[14] E. O. Olaniyi, O. K. Oyedotun, and K. Adnan, Heart diseases diagnosis using neural networks arbitration, Int. J. Intell. Syst. Appl., vol. 7, no. 12, p. 72, 2015.

[15] Das, I. Turkoglu, and A. Sengur, Effective diagnosis of heart disease through neural networks ensembles, Expert Syst. Appl., vol. 36, no. 4, pp. 7675_7680, May 2009.

[16] O. W. Samuel, G. M. Asogbon, A. K. Sangaiah, P. Fang, and G. Li, An integrated decision support system based on ANN and Fuzzy_AHP for heart failure risk prediction,Expert Syst. Appl., vol. 68, pp. 163_172, Feb. 2017.

[17] S. I. Ansarullah and P. Kumar, A systematic literature review on cardiovascular disorder identication using knowledge mining and machine learning method, Int. J. Recent Technol. Eng., vol. 7, no. 6S,pp. 1009_1015, 2019.

[18] S.Hema Kumar, J.UdayKiran, V.D.AKumar, G.Saranya, Ramalakshmi V.Effective Online Medical Appointment System. International Journal of Scientific \& Technology Research, Volume 8, Issue 09, September 2019, Pages 803 - 805.

[19] V.D.Ambeth Kumar, G.Gokul, S.Malathi, K.Vengatesan, D.Elangovan, B.Chitra.Implementation Of The Pulse Rhythemic Rate For The Efficient Diagonising Of The Heart Beat. Healthcare Technology Letters (IET) 2019 Apr 17;6(2):48-52.

[20] S.V. Ruphitha et.al.Management of Major Postpartum Haemorrhage by using Zigbee protocol - A Review . 2021 6th International Conference on Inventive Computation Technologies (ICICT) (DOI: 10.1109/ICICT50816.2021.9358757)

[21] Ambeth Kumar.V.D, Dr.M.Ramakrishnan, V.D.Ashok Kumar and Dr.S.Malathi (2015) . Performance Improvement using an Automation System for Recognition of Multiple Parametric Features based on Human Footprint. kuwait journal of science, Vol 42, No 1 (2015), pp:109-132.

[22] Ramakrishan.M et.al..Footprint Based Recognition System. in the month of April for the International Journal Communication in Computer and Information System (CCIS) Journal (Springer) Volume 147, Part 3, 358-367, DOI: 10.1007/978-3-642-20573-6_63, April 2011

[23] Ramya,T., .Malathi,S.,ratheeksha,G.R. and V.D.Ambeth Kumar (2014).Personalized authentication procedure for restricted web service access in mobile phones. Applications of Digital Information and Web Technologies (ICADIWT), 2014, Page(s):69 - 74, Bangalore, India (ISBN:978-1-4799-2258-1)

[24] Indhumathi.M et.al .Healthcare Management of Major Cardiovascular Disease-A review.2021 6th International Conference on Inventive Computation Technologies (ICICT), (DOI: 10.1109/ICICT50816.2021.9358519)

[25] Ambeth Kumar.V.D (2018). Novel Wireless Sensing System For The Welfare Of Sewer Laborers.Healthcare Technology Letters (IET) Volume 5, Issue 4, p. 107 -112. 